\title{
Clinicians' experiences of using and implementing a medical mobile phone app (QUiPP V2) designed to predict the risk of preterm birth and aid clinical decision making
}

\author{
N. Carlisle ${ }^{*} \mathbb{D}$, H. A. Watson ${ }^{*+}$, J. Carter, K. Kuhrt, P. T. Seed, R. M. Tribe, J. Sandall and A. H. Shennan
}

\begin{abstract}
Background: As the vast majority of women who present in threatened preterm labour (TPTL) will not deliver early, clinicians need to balance the risks of over-medicalising the majority of women, against the potential risk of preterm delivery for those discharged home. The QUiPP app is a free, validated app which can support clinical decision-making as it produces individualised risks of delivery within relevant timeframes. Recent evidence has highlighted that clinicians would welcome a decision-support tool that accurately predicts preterm birth.

Methods: Qualitative interviews were undertaken as part of the EQUIPTT study (The Evaluation of the QUiPP app for Triage and Transfer) (REC: 17/LO/1802) which aimed to evaluate the impact of the QUiPP app on management of TPTL. Individual semi-structured telephone interviews were used to explore clinicians' (obstetricians' and midwives') experiences of using the QUiPP app and how it was implemented at their hospital sites. Thematic analysis was chosen to explore the meaning of the data, through a framework approach.
\end{abstract}

Results: Nineteen participants from 10 hospital sites in England took part. Data analysis revealed three overarching themes which were: 'experience of using the app', 'how QUiPP risk changes practice' and 'successfully adopting QUiPP: context is everything.' With these final themes we appeared to have achieved our aim of exploring the clinicians' experiences of using and implementing the QUiPP app.

Conclusion: This study explored different clinician's experiences of implementing the app. The organizational and cultural context at different sites appeared to have a large impact on how well the QUiPP app was implemented. Future work needs to be undertaken to understand how best to embed the intervention within different settings. This will inform scale up of QUiPP app use across the UK and ensure that clinicians have access to this free, easy-to-use tool which can positively aid clinical decision making when caring for women in TPTL.

Clinical trial registry and registration number: ISRCTN 17846337, registered 08th January 2018, https://doi.org/10.1186/ ISRCTN17846337.

\footnotetext{
*Correspondence: naomi.h.carlisle@kcl.ac.uk; helenawatson85@gmail.com

${ }^{\dagger} \mathrm{N}$. Carlisle and H. A. Watson co-first authors for this work

Department of Women and Children's Health, School of Life Course

Sciences, King's College London, St Thomas' Hospital, 10th Floor North

Wing, Westminster Bridge Road, London SE1 7EH, UK
}

(C) The Author(s) 2021. Open Access This article is licensed under a Creative Commons Attribution 4.0 International License, which permits use, sharing, adaptation, distribution and reproduction in any medium or format, as long as you give appropriate credit to the original author(s) and the source, provide a link to the Creative Commons licence, and indicate if changes were made. The images or other third party material in this article are included in the article's Creative Commons licence, unless indicated otherwise in a credit line to the material. If material is not included in the article's Creative Commons licence and your intended use is not permitted by statutory regulation or exceeds the permitted use, you will need to obtain permission directly from the copyright holder. To view a copy of this licence, visit http://creativecommons.org/licenses/by/4.0/. The Creative Commons Public Domain Dedication waiver (http://creativeco mmons.org/publicdomain/zero/1.0/) applies to the data made available in this article, unless otherwise stated in a credit line to the data. 
Keywords: Preterm birth, App, QUiPP, Threatened preterm labour, Decision-making

\section{Background}

It is difficult for clinicians to accurately determine which women with threatened preterm labour (TPTL) are at true risk of preterm birth. As the vast majority of women who present in TPTL will not deliver early, clinicians need to balance the risks of over-medicalising the majority of women, against the potential risk of preterm delivery for those discharged home [1]. When managing the risks of preterm birth, clinicians may prefer to 'err on the side of caution' [2], an attitude which appears to be reflected in the current preterm birth guidance in England [3]. However less wary clinicians are cautious of over-medicalisation [4] and as a result there are now wide variations in practice [5].

The QUiPP app is a free, validated app which can support clinical decision-making as it produces individualised risks of delivery within relevant timeframes [6-9]. The current version (Version 2) of the QUiPP predictor (for symptomatic women arriving in threatened preterm labour) is based on over 1000 women [9]. The risk score it produces is based upon the presence or absence of major risk factors, her clinical quantitative fetal fibronectin (qfFN) result and/or transvaginal ultrasound measurement of cervical length (CL). It has recently been recommended by NHS England [10], and a toolkit for implementation in hospital sites has been produced [11]

Decision support which includes a risk score with guidance, such as a cut-off for intervention, may be useful. Findings from our Delphi survey of preterm birth specialists suggested that when women arrive in TPTL, a QUiPP app score of $\geq 5 \%$ risk of delivery within a week could help guide who should be admitted [12]. The QUiPP app can also display the risk score in an infographic donut, allowing clinicians to better communicate with women who prefer visual representations of risk (Fig. 1).

There is complicated and delicate reasoning that occurs behind obstetric decision-making [13] and recent evidence has highlighted that clinicians would welcome a decision-support tool that accurately predicts preterm birth [2].

As digital technology becomes more popular in healthcare, and in maternity care [14], there are concerns that utilising this technology to triage could reduce the likelihood of patients being assessed holistically [15]. Specific qualitative research on clinicians' decision making when caring for women with TPTL is lacking. This paper reports findings of a study that explored clinicians' experiences of using and implementing the QUiPP app in clinical practice in England.

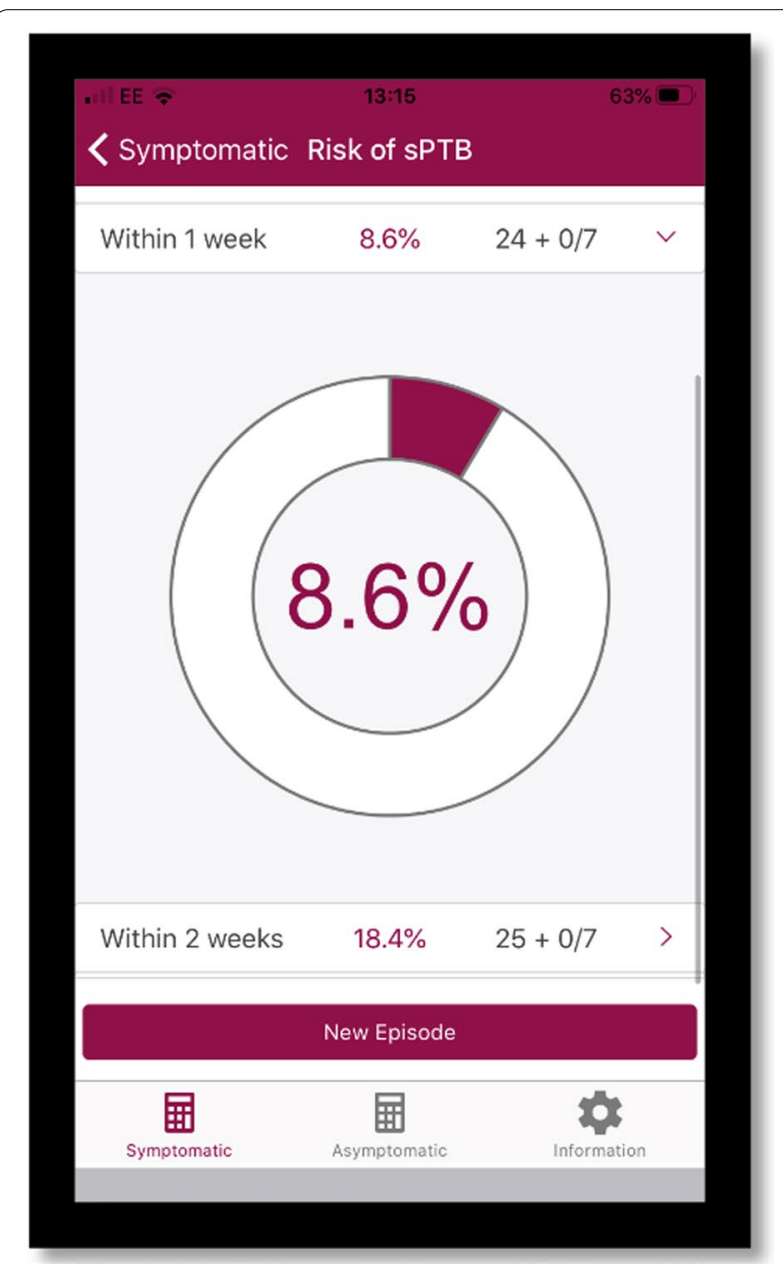

Fig. 1 An image generated using the QUiPP app and knowledge of risk factors, cervical length and fetal fibronectin measurement. *Risk is displayed as a donut risk infographic. In this example, a woman has an $8.6 \%$ risk of delving with 1 week of the test and a $18.4 \%$ risk of delivering within 2 weeks. When clicking on the risk score, the donut risk infographic appears below. Image used with consent from the QUiPP Team

\section{Methods}

Study design

Qualitative interviews were undertaken as part of the EQUIPTT study (The Evaluation of the QUiPP app for Triage and Transfer) (REC: 17/LO/1802) [16-18] which aimed to evaluate the impact of the QUiPP app on management of TPTL. The interviews were used to explore clinicians' experiences of using the QUiPP app and how it was implemented at their hospital sites.

The study was carried out with participants from 10 of the 13 EQUIPTT study hospital sites, (geographically 
situated in three areas across England: London, the South East of England, and the Midlands area of England). Clinicians (midwives and obstetric doctors) were eligible if they had experience of the QUiPP app as part of TPTL management during the EQUIPTT trial. In England, while obstetricians, not midwives, make management of care decisions regarding women in TPTL, it is midwives who first assess women when they arrive to hospital. Furthermore, one hospital in this study worked under their local guidance which permitted midwives to undertake speculums and qfFN swabs on women under 37 weeks' gestation, meaning the midwives are undertaking the predictive test required for input in the QUiPP app. This provides obvious benefits with time efficiency when triaging women, and we are aware that undertaking this change to local guidance is gaining traction with other hospitals across the United Kingdom. Midwives are therefore welcome to use the app and encouraged to. This also provides the benefit of promoting multidisciplinary decision making and cohesion which is important to promote in light of the recent Ockenden Report [19] (a report reviewing failings in maternity care in England).

The Framework approach was utilised [20], which is a systematic model of qualitative data analysis that is widely used in health research [21]. Purposive sampling was undertaken, ensuring the sample included clinicians who worked in both tertiary and secondary care settings, midwives and obstetricians, and junior and senior staff. This sample also included those who oversaw the research project at their unit with knowledge of the app but who were not using it directly in clinical care (showing or advising others to use the app rather than directly using it on women with TPTL symptoms themselves). This meant that the views of the multi-disciplinary team could be gathered, and a diversity of perspectives explored.

Potential clinicians were identified through sending an invitation by email to the principal investigators and research midwives at the 13 EQUIPTT sites who forwarded the invitation to their colleagues. Interested clinicians were given a Participant Information Leaflet and had the opportunity to ask any questions. Informed written consent was obtained before the interview was conducted via telephone at the participant's convenience.

\section{Data collection}

All clinician interviews were conducted between January and March 2019 via telephone and recorded with consent on encrypted digital audio equipment. As recordings were made there were no field notes. The recordings were then uploaded onto a secure, password protected University approved computer. Data collection and handling was General Data Protection Regulation (GDPR) compliant.

The semi-structured interview schedule was designed by two researchers (NC and $\mathrm{HW}$ ) who have both clinical and research experience. This ensured it was robust enough to meet the aims of the study while ensuring clinical significance. Designing the schedule included building on previous research findings in this area and ensuring prompts/questions were included that covered the main aims and issues that this study was trying to achieve. Prompts were used to encourage expansion on certain points and encourage more detailed discussion on topics as they arose. Interview techniques aimed to build rapport [22] by active listening, summarising responses to check understanding and rephrasing of questions which were not fully answered the first time. As the participants were busy clinicians, we ensured that the interviews lasted no longer than $45 \mathrm{~min}$. Most of the interviews took 20-30 min.

The interview schedule covered the background of the participant (their role, how long they had been involved in making decisions around the care of women with TPTL, and if they worked in a district general or tertiary unit), their knowledge and experience of the QUiPP app, and their opinions and values on its use.

In order to reduce researcher bias, the participants who were midwives had their interviews undertaken by a researcher who was also an obstetrician $(\mathrm{HW})$, and obstetricians were interviewed by a researcher who was also a midwife (NC).

\section{Data analysis}

The interview recordings were transcribed by a third party. Data were anonymised and given a study identification number. Two researchers (NC and HW) then checked the typed transcriptions for accuracy relative to the recordings.

Firstly, the data were indexed, and participants' characteristics were identified (if they were a midwife or obstetrician, and if they worked in a tertiary or district general unit). Thematic analysis was chosen to explore the meaning of the data, through a framework approach [23, 24]. The contents of the transcripts were analysed by the two researchers and coded according to emergent themes. After listening to and reading the transcripts several times, a coding scheme was developed using the software NVivo 12 Pro [25]. Constant communication between the two researchers ensured they corroborated on the themes that were being produced. Utilising two researchers ensured that interpretation bias was minimised.

The coding scheme's descriptive labels were applied to the raw data in the transcripts. This coding scheme was then progressively refined into key themes which 
reflected the key research questions and allowed comparisons within and between cases. In order to understand and attempt to explain the patterns between these classified experiences, a case comparative model was adopted to explore the contextual conditions which may be associated with our findings [26].

\section{Reflexivity}

As NC and HW are researchers and clinicians who were involved with the development and evaluation of the QUiPP app and coordination of the EQUIPTT trial, it was essential that bias was minimised when analysing the data. Reflexive sensitivity was required to ensure the researchers existing theories about the utility of the QUiPP app were not simply corroborated. Negative, unexpected and conflicting findings were actively sought, examined in detail [27] and have been highlighted in the discussion section of this manuscript. If any clinical concerns were noted during the interview these would have been raised with the individual clinicians and sites directly to avoid these clinical findings over-shadowing exploration of the interviewees' experiences.

\section{Findings}

Nineteen participants gave their consent and took part in individual semi-structured telephone interviews (Table 1). As highlighted in the table, some participants were not involved in direct clinical care during the trial and were responsible for research delivery (showing or advising others to use the app) at their local hospital. However, none of the participants have been involved in the development of the QUiPP app, or were part of the co-ordinating research team for the EQUIPTT trial.

Data analysis revealed three overarching themes which were: 'experience of using the app', 'how QUiPP risk changes practice' and 'successfully adopting QUiPP: context is everything. With these final themes we appeared to have achieved our aim of exploring the clinicians' experiences of using and implementing the QUiPP app. Verbatim quotes have been included within the themes, using the participant numbers seen in Table 1.

\section{Theme one: experience of using the app \\ An accessible and acceptable tool}

Clinicians appreciated that the QUiPP app was accessible, and its availability on a smart phone was perceived positively in terms of being quick and easy to use, especially in the busy acute hospital setting. "...it's hard to get

Table 1 Table to describe the EQUIPTT clinician interview study participants

\begin{tabular}{|c|c|c|}
\hline Participant number & Job title & Hospital site \\
\hline 1 & $\begin{array}{l}\text { Research midwife (a registered midwife with clinical experience who ensures research deliv- } \\
\text { ery and recruitment at their local hospital) }\end{array}$ & Tertiary \\
\hline 2 & Research midwife ${ }^{a}$ & $\begin{array}{l}\text { DGH (District } \\
\text { General Hos- } \\
\text { pital) }\end{array}$ \\
\hline 3 & Research midwife ${ }^{a}$ & Tertiary \\
\hline 4 & Research midwife ${ }^{a}$ & DGH \\
\hline 5 & Research midwife ${ }^{a}$ & DGH \\
\hline 6 & Consultant obstetrician ${ }^{b}$ & DGH \\
\hline 7 & Consultant obstetrician ${ }^{b}$ & DGH \\
\hline 8 & MAU (Maternity Assessment Unit or Triage) coordinating midwife & DGH \\
\hline 9 & MAU midwife ${ }^{b}$ & DGH \\
\hline 10 & MAU midwife ${ }^{b}$ & DGH \\
\hline 11 & Consultant obstetrician ${ }^{b}$ & DGH \\
\hline 12 & Research midwife ${ }^{a}$ & DGH \\
\hline 13 & Consultant obstetrician ${ }^{b}$ & DGH \\
\hline 14 & ST3 obstetrics ${ }^{b}$ & DGH \\
\hline 15 & Consultant obstetrician ${ }^{b}$ & DGH \\
\hline 16 & MAU midwife ${ }^{b}$ & Tertiary \\
\hline 17 & Research midwife ${ }^{a}$ & DGH \\
\hline 18 & Speciality obstetric registrar ${ }^{b}$ & Tertiary \\
\hline 19 & Consultant obstetrician ${ }^{\mathrm{b}}$ & Tertiary \\
\hline
\end{tabular}

${ }^{a}$ Not involved in direct clinical care during the trial

${ }^{\mathrm{b}}$ Involved in direct clinical care during the trial 
on the computers. So the fact that we can use our phones means that you don't get slowed down." [P16]. Clinicians also liked that the app was "easy to access" [P13] with a simple and straight-forward user interface. Such features were felt to help integrate QUiPP's use into individual practice with ease. "From the moment I downloaded the app and I used for the first time I kept using for every single patient." [P18]. Acceptability was perceived to be higher among younger users: "It was very easy to do because most of the juniors are very happy downloading anything that's going to happen onto their phone" [P11] with a junior doctor commenting how "I use my smart phone for everything at work so I just think it's easy" [P14].

However, initial resistance to the innovation was noted. “...some people are a bit funny about 'oh I don't want to download it onto my phone.' I think once they then did it, they saw that it wasn't an issue and it was fine." [P1]. One senior clinician highlighted that the correct use of the app may be impaired by the experience of the user. Some junior clinicians may not fully understand or misinterpret the obstetric definitions in the app fields (for example, cervical surgery does not include punch biopsy, and previous preterm birth does not include iatrogenic deliveries [e.g. if a woman was induced at 32 weeks for pre-eclampsia]): “...it's potentially misinterpretation... based on the inexperience of the doctors." [P6].

\section{App threshold felt right}

The $5 \%$ risk of delivery within seven days was intended as guidance only, but it was important to explore how this was perceived by our clinician stakeholders in actual use. Clinicians agreed that they liked using the 5\% threshold as a guide to decision-making, with one research midwife responsible for training clinicians in using the app saying: "I think the $5 \%$ is quite comfortable." [P3].

Clinicians involved in direct clinical care also expressed trust and belief in the risk scores given by the app, with one participant noting how there is: "... a big trust in the app [P10]", and another participant feeling "I think that people...did trust" the app [P9].

\section{Supportive tool not a clinical crutch}

Understanding the QUiPP App as a supportive clinical tool, rather than a dogmatic diagnostic tool proved challenging. When explaining the app to colleagues, clinicians had to: "really push them to see past that $5 \%$ and actually see that it was just a guidance" [P1], reminding them that the QUiPP app: “...is a tool and it shouldn't override your clinical judgement" [P18]. Examples were provided of clinicians using their judgement alongside the app to provide woman-centred care: "...a couple of times I have admitted them...despite the risk being lower than $5 \%$...probably only in 2 cases, very anxious patients which usually comes together with a very bad history and anxiety." [P18]. The interviewee's use of 'despite' in the phrase "despite the risk being less than 5\%" suggests a lack of awareness that this use reflects the intended QUiPP use, rather than a breach of use.

\section{Theme two: how QUiPP risk changes practice Reconsidering risk}

Clinicians found that the QUiPP app risk scores made them reconsider their perception of risk. For most clinicians, the scores adjusted their perceptions of risk to a lower level: "...most women actually have a very low risk of going into labour early compared to just saying 'positive fibronectin"' [P3].

The percentage risk scores prevented them from categorising the woman into a binary outcome (to admit or discharge). This compelled them to be more analytical in their assessment and management plan:

Previous[ly]...you wouldn't particularly think whether the patient was likely to deliver or not, you would just think admission or not. Whereas now there is an actual risk, so I think you can personalise the care a bit better. [P3].

Personalised care was also aided by the long-term percentage risks which are also produced by the QUiPP app, in addition to the risk of delivery within one week. This allows for tailored follow-up plans: "...it's not just about that $5 \%$ but it's about the other percentages as well." [P1].

This suggests that there is a professional development aspect to using the QUiPP app as it allows clinicians to critically think about preterm labour risk, and how they perceive and manage TPTL.

However, some clinicians thought that utilising the long-term percentage risks "opened up a bit of a can of worms" [P14] in busy acute settings, despite viewing the short-term percentage risks as favourable. The additional long-term information is impractical if clinicians have no pathway that they can offer these women, such as a preterm birth clinic. Meanwhile the short-term risk does not prevent them from admitting or discharging women in the acute setting.

\section{Confidence in clinical decisions}

Not all interviewees felt their risk perception changed but QUiPP's validation of their existing judgement was still valued: “...it doesn't really tell you anything you don't already kind of know in your own head...it's just trying to put a number on what your own thought process." [P6].

Presenting the same information in a straight-forward percentage format: "structures their thought process" [P5]. This increased clinicians' confidence in their clinical 
decision making as both doctors and midwives felt they had: "an extra layer of prediction into your clinical acumen" [P19].

\section{"Better conversations" with women}

Clinicians found that they had "better conversations" [P6] with women as a result of the QUiPP app. This was because: "women understand percentages" [P16] compared to presenting a fetal fibronectin (fFN) result (often quantitatively in unfamiliar and complicated $\mathrm{ng} / \mathrm{ml}$ ) or a cervical length measurement.

Whilst some clinicians were not aware of the donut infographics (Fig. 1), others found they aided explanations of risk to women:“...I always say to them, you know, $10 \%$ risk of delivery before 37 weeks, actually that means that you've got a $90 \%$ deliver after and that's where your donuts come in" [P19].

One interviewee also explained how QUiPP's longterm risk scores aid shared-decision making about returning with further symptoms: "...to the woman...you might say 'well the risks straight away is quite low so you can go home, but it does sort of suggest you might have a risk so come back if you're worried'. I mean you always say come back if you're worried anyway, but I can use that to also help in counselling." [P11].

Clinicians also highlighted the ability of women being able to download the app themselves; empowering them to be involved in their own health, care plan and decision making: "I like it that the patients can download it themselves... physically seeing the numbers on...their own phone." [P13].

\section{Theme three: successfully adopting QUiPP: context is everything}

\section{Time is of the essence}

The majority of participants felt the QUiPP app improved the use of their time and reduced the cascade of unnecessary intervention. "It's a tool that potentially can...reduce our workload...especially if we can reduce the amount of, um, steroids and potentially sliding scale in this use." [P3].

The app was sometimes seen as "something extra" [P1] and "to start with 'oh something else we've got to do"' [P2]. However often clinicians thought "it was going to take more time than it actually did" [P12]. Some did not mind even if it did take more time: "I don't mind taking a bit [more] time if I was giving better care." [P16].

\section{Training and retaining clinicians}

New clinicians quickly became engaged in using the QUiPP app, especially in sites were the app was already implemented well: "... when we had new staff come into the unit, sort of rotating round ... that sort of enthusiasm would rub off on them....and helped them to engage in using it as well." [P6].

If enthusiasm was lacking with current staff, clinicians found that this changed when new staff started as they brought "a bit of a renewed energy" [P4].

Staffing pressures were noted at nearly all the sites, which affected how successfully the QUiPP app was adopted. For example, clinicians highlighted that frequent utilisation of locums instead of regular staff hindered implementation of the app. Clinicians also discussed how working in a busy, short-staffed unit meant that additional skills (such as cervical length scanning) are not taught: "We get junior doctors and trainees who... are great in triage, but obviously they require training for the cervical length and there is a high turnover...the cervical length training takes a while."[P3]. If clinicians are not taught how to undertake a cervical length, then they can only ever use the QUiPP app on the qfFN function.

\section{Conflicting attitudes towards change}

Whilst staffing shortages hindered utilisation, more than one site mentioned a lack of cohesion among the consultant body which impaired implementation. Participants also thought that junior clinicians were the most enthusiastic adopters, while some senior clinicians seemed sceptical: "I think sometimes it's just a general 'oh, you know, we've been doing it this way for a long time so, you know, we don't necessarily want to try something new', or ... um, 'I trust my experience more"' [P4].

Participants felt that having positive senior colleagues or consultants who repeatedly endorsed the app helped implementation. Other clinicians felt this endorsement could be from any member of the multi-disciplinary team "there are like these two midwives that [have]...taken that as their responsibility, it's definitely made a big difference." [P10]. This demonstrates that if someone was willing to take on ownership and responsibility for the app, they could have a positive impact on the whole multidisciplinary team, despite not being the most senior clinician. In some units midwives expressed the view that using QUiPP was the doctor's responsibility alone, while other midwives felt it was their responsibility to "encourage them" [P1] to use the app. This may reveal as much about interdisciplinary cohesion as QUiPP itself, but the successful adoption of the app is likely to require engagement and ownership from all stakeholders in preterm labour triage, not just the clinicians who input the clinical data into the app.

The culture towards innovation within their department also had a large effect on implementation of the app. Some units seemed to have a negative attitude towards change: "Honestly people just don't care...Why would they care? They just want to get through their 
shift." [P14]. Other units were more positive: "as a unit I think we are very ... are very open to change and development and improvement to patients" [P6]. One site implemented both fFN and the QUiPP app at the same time and reported a very positive attitude to using the QUiPP app. This may have been because at this site, the QUiPP approach to risk did not need to supplant established preconceptions around binary (positive or negative) fFN values.

\section{Discussion}

In this study we interviewed 19 clinicians from 10 hospital sites across three areas in England (London, the South East of England, and the Midlands area of England). This is the first study to explore the experiences of clinicians using a predictive tool to support clinical decision making in care of women with TPTL. The findings also provided insights into how the QUiPP app was implemented in different hospital sites.

Limitations of this study include how participants were not given the opportunity to review the transcripts or findings. Some hospitals had higher workloads than others, meaning clinicians were unable to be interviewed, leading to representation from only 10 of the 13 sites involved in the EQUIPTT study. However, we feel that we achieved representation from a diverse range of sites with varying birth rates, a mix of district general and tertiary units and a range of clinical backgrounds. While participants were not part of the co-ordinating research team or involved in the development of the app, some clinicians interviewed were involved in overseeing research at their local hospital site.

Clinicians appear to find the QUiPP app an accessible and acceptable clinical tool in the triage process, that QUiPP app scores changed how they perceived TPTL risk and increased their confidence in clinical decision making. Clinicians also felt the QUiPP scores allowed them to have better conversations with women they cared for. Given what is known about the uncertainty and conflict women experience during TPTL and how interactions with professionals impact this stressful experience [28], these findings support the use of QUiPP in this setting.

While most clinicians felt the QUiPP app gave them more confidence in their clinical decision making and enhanced communication with women, some participants found it challenging to view the QUiPP app as a supportive but non-dogmatic tool. Concerns have been raised that technology triage tools can prevent holistically assessment [15], which highlights the importance of educating and reiterating that the QUiPP app is a tool to support clinical decision making when implementing into hospital sites.
Some participants felt that the app may be used incorrectly due to misinterpreting the obstetric definitions in the app fields. Whilst an information section is available within the app which contains a detailed guide to the field definitions, this was clearly not always utilised. Therefore, future versions of the app will ensure that the user guide is more accessible and/or the app fields are clearer. Other feedback included requests for determining whether, with more data, there are additional risk factors that could be included in the app, as well as including confidence intervals when the app produces a result. Whether adjustments are made to the app itself, or in how the app is implemented at sites, requires further study.

These interviews revealed that some sites did not utilise the long-term preterm birth risks that the app provides. This may be because they do not currently have a mechanism to care for these women who have a low short-term risk, but a higher long-term risk. As the recently published Saving Babies Lives Care Bundle Version 2 [29] is applied across England, it is hoped that preterm birth clinic provision will improve and the pathway for women more established.

The ease of QUiPP app implementation varied between hospital sites. Our interviews did not suggest that these difference in implementation were associated with type of hospital unit or clinician qualification. Yet staff shortages, busy acute settings and hospital culture all seemed to affect the degree of success. However, once clinicians started using the app, the majority felt it improved the use of their time. As service providers strive to encourage a workplace culture which is open to change, learning and improvement, future implementation efforts for QUiPP will need to reconsider how to deliver training to this dynamic workforce and embed this innovation within existing practices.

The increasing need for a preterm birth clinical decision tool for clinicians has been recognized [2]. This study demonstrates that the majority of clinicians find the QUiPP app accessible and helpful in clinical decision making [14]. However, our findings suggest a need to highlight that QUiPP is a clinical decision support tool, rather than a diagnostic tool, and that the app field definitions may need clarification. These are two important areas that require emphasis when implementing the app in new hospital sites.

\section{Conclusions}

This study explored different clinician's experiences of implementing the app in England. The organizational and cultural context at different sites appeared to have a large impact on how well the QUiPP app was implemented. Future work needs to be undertaken to understand how best to embed the intervention within different settings. 
This will inform scale up of QUiPP app use across the UK and ensure that clinicians have access to this free, easy-to-use tool which can positively aid clinical decision making when caring for women in TPTL.

\author{
Abbreviations \\ TPTL: Threatened preterm labour; qfFN: Quantitative fetal fibronectin; CL: \\ Cervical length; EQUIPTT study: The Evaluation of the QUiPP app for Triage \\ and Transfer.
}

\section{Acknowledgements}

Not applicable.

\section{Authors' contributions}

AHS, HAW and JC conceived and designed the study. HAW and AHS drafted the original grant proposal and trial protocol with input from PS, RMT and JC. NC, KK, HAW and AHS provided day to day oversight of participant recruitment and day to day running of the trial. NC and HAW undertook the qualitative interview data collection. NC and HAW analysed the data with oversight from JC and JS. NC drafted the manuscript with additional input from HAW and JS. All authors read and approved the final manuscript.

\section{Funding}

The development of the QUiPP app and the EQUIPTT study are funded by the Guy's and St Thomas' Charity (Registered Charity No. 1160316) and Tommy's (1060508). As it is a portfolio study, recruitment is supported through local Clinical Research Networks. King's College London and Guy's and St Thomas' NHS Foundation Trust are co-sponsors of the study but neither sponsor provided funding for the trial. The development of the QUiPP app and the EQUIPTT study are funded by the Guy's and St Thomas' Charity (Registered Charity No. 1160316) and Tommy's (1060508). This work is supported by the clinical research network led by South London, the Biomedical Research Centre at Guy's and St Thomas' NHS Foundation Trust and King's College London. HW is funded by a Guy's and St Thomas' NHS Trust Biomedical Research Centre Clinical Training Fellowship. PTS is partly funded by Tommy's (Registered Charity No. 1060508) and by NIHR Applied Research Collaboration South London (NIHR ARC South London). JC was funded by the National Institute for Health Research's NIHR/HEE CAT Clinical Doctoral Research Fellowship Programme (Ref. CDRF-2013-04-026). JS is an NIHR Senior Investigator and supported ARC by the National Institute for Health Research (NIHR) Applied Research Collaboration South London (NIHR ARC South London) at King's College Hospital NHS Foundation Trust. AHS and RMT receive funding from the NIHR Biomedical Research Centre based at Guy's and St Thomas' NHS Foundation Trust and King's College London. The views expressed are those of the authors and not necessarily those of the NHS, the NIHR or the Department of Health and Social Care.

\section{Availability of data and materials}

The datasets generated and/or analysed during the current study are not publicly available but are available from the corresponding author on reasonable request.

\section{Declarations}

\section{Ethical approval and consent to participate}

EQUIPTT was granted a favourable ethical opinion (REC reference 17/LO/1802) by the London Bridge Research Ethics Committee on 21 November 2017. Informed written consent was obtained from all participants.

\section{Consent for publication}

Not applicable.

\section{Competing interests}

AS is Principal Investigator on Hologic ${ }^{\circledR}$ funded science grants, which are paid directly to institute. $\mathrm{NC}$ received financial assistance from Hologic ${ }^{\circledR}$ covering expenses only, paid directly to institute, to provide educational talks on preterm birth. The other authors report no conflicts of interest.
Received: 9 October 2020 Accepted: 3 November 2021

Published online: 18 November 2021

\section{References}

1. DeFranco EA, Lewis DF, Odibo AO. Improving the screening accuracy for preterm labor: is the combination of fetal fibronectin and cervical length in symptomatic patients a useful predictor of preterm birth? A systematic review. Am J Obstet Gynecol. 2013;208:233.e1-233.e6. https://doi.org/10. 1016/J.AJOG.2012.12.015

2. White H, Morton VH, Stock SJ, Lavender T. Preterm labour decision-making and experiences of care for women and clinicians (QUIDS Qualitative): a qualitative exploration. Sex Reprod Healthc. 2019;21:95-101. https://doi. org/10.1016/J.SRHC.2019.06.005.

3. NICE. Preterm labour and birth. London: Natl Inst Heal Care Excell; 2015.

4. Watson H, Re RA. Preterm labour: summary of NICE guidance. BMJ Br Med J. 2015. https://www.bmj.com/content/351/bmj.h6283/rr.

5. Care A, Ingleby L, Alfirevic Z, Sharp A. The influence of the introduction of national guidelines on preterm birth prevention practice: UK experience. BJOG Int J Obstet Gynaecol. 2019. https://doi.org/10.1111/1471-0528. 15549.

6. Kuhrt K, Smout E, Hezelgrave N, Seed PT, Carter J, Shennan AH. Development and validation of a tool incorporating cervical length and quantitative fetal fibronectin to predict spontaneous preterm birth in asymptomatic high-risk women. Ultrasound Obstet Gynecol. 2016;47:104-9.

7. Watson HA, Carter J, Seed PT, Tribe RM, Shennan AH. The QUIPP app: a safe alternative to a treat-all strategy for threatened preterm labour. Ultrasound Obstet Gynecol. 2017;50:342-6.

8. Watson HA, Seed PT, Carter J, Hezelgrave NL, Kuhrt K, Tribe RM, et al. Development and validation of the predictive models for the QUiPP App v.2: a tool for predicting preterm birth in high-risk asymptomatic women. Ultrasound Obstet Gynecol. 2019. https://doi.org/10.1002/uog.20401.

9. Carter J, Seed PT, Watson HA, David AL, Sandall J, Shennan AH, et al. Development and validation of prediction models for the QUiPP App v.2: a tool for predicting preterm birth in women with symptoms of threatened preterm labor. Ultrasound Obstet Gynecol. 2019. https://doi.org/10. 1002/uog.20422.

10. NHS England. Appendix I: implications of COVID-19 on reducing preterm births. 2020. https://www.england.nhs.uk/wp-content/uploads/2020/04/ C0499-Appx-I-to-SBLCBv2-Reducing-preterm-births.pdf.

11. Carlisle N, Watson HA, Shennan AH. Development and rapid rollout of The QUiPP App Toolkit for women who arrive in threatened preterm labour. BMJ Open Qual. 2021;10:e001272. https://doi.org/10.1136/ bmjoq-2020-001272.

12. Carter J, Tribe R, Watson H, Shenann A. Threatened preterm labour management: results of a Delphi consensus on best practice (poster presentation). BJOG Int J Obstet Gynaecol. 2016;123:100-1.

13. Diamond-Brown L. "It can be challenging, it can be scary, it can be gratifying": obstetricians' narratives of negotiating patient choice, clinical experience, and standards of care in decision-making. Soc Sci Med. 2018;205:48-54. https://doi.org/10.1016/J.SOCSCIMED.2018.04.002.

14. Carter J, Sandall J, Shennan AH, Tribe RM. Mobile phone apps for clinical decision support in pregnancy: a scoping review. BMC Med Inform Decis Mak. 2019;19:219. https://doi.org/10.1186/s12911-019-0954-1.

15. Castle-Clarke S, Imison C. The digital patient: transforming primary care? 2016. https://www.nuffieldtrust.org.uk/files/2019-08/nt-the-digital-patie nt-web-update-august-2019.pdf.

16. Watson HA, Carlisle N, Kuhrt K, Tribe RM, Carter J, Seed P, et al. EQUIPTT: the evaluation of the QUiPP app for Triage and Transfer protocol for a cluster randomised trial to evaluate the impact of the QUiPP app on inappropriate management for threatened preterm labour. BMC Pregnancy Childbirth. 2019;19:68. https://doi.org/10.1186/s12884-019-2210-1.

17. Watson HA, Carlisle N, Seed PT, Carter J, Kuhrt K, Tribe RM, et al. Evaluating the use of the QUiPP app and its impact on the management of threatened preterm labour: a cluster randomised trial. PLoS Med. 2021;18: e1003689. https://doi.org/10.1371/journal.pmed.1003689.

18. Carlisle N, Watson HA, Kuhrt K, Carter J, Seed PT, Tribe RM, et al. Ten women's decision-making experiences in threatened preterm labour: qualitative findings from the EQUIPTT trial. Sex Reprod Healthc. 2021. https://doi.org/10.1016/j.srhc.2021.100611. 
19. Department of Health and Social Care. Ockenden review of maternity services at Shrewsbury and Telford Hospital NHS Trust. 2020. https:// www.gov.uk/government/publications/ockenden-review-of-maternityservices-at-shrewsbury-and-telford-hospital-nhs-trust.

20. Ritchie J, Lewis J. Qualitative research practice: a guide for social science students \& researchers. Thousand Oaks: Sage; 2003.

21. Gale NK, Heath G, Cameron E, Rashid S, Redwood S. Using the framework method for the analysis of qualitative data in multi-disciplinary health research. BMC Med Res Methodol. 2013;13:117. https://doi.org/10.1186/ 1471-2288-13-117.

22. Drabble L, Trocki KF, Salcedo B, Walker PC, Korcha RA. Conducting qualitative interviews by telephone: lessons learned from a study of alcohol use among sexual minority and heterosexual women. Qual Soc Work. 2016;15:118-33

23. Ormston R, Spencer $L$, Barnard M, Snape $D$. The foundation of qualitative research. In: Ritchie J, Lewis J, Nicholls CM, Ormston R, editors. Qualitative Research Practice A Guide for Social Science Students and Researchers. 2013. pp. 1-27.

24. Hackett A, Strickland K. Using the framework approach to analyse qualitative data: a worked example. Nurse Res. 2018;26:8-13.
25. QSR International. NVivo 12 Pro. 2018. https://www.qsrinternational.com/ nvivo/nvivo-products/nvivo-12-pro.

26. Spencer L, Ritchie J, O'Conner W. Analysis: practices, principles and processes. In: Ritchie J, Lewis J, editors. Qualitative research practice: a guide for social science students and researchers. Sage Limited. 2003.

27. Yardley L. Dilemmas in qualitative health research. Psychol Health. 2000;15:215-28.

28. Carter J, Tribe RM, Shennan AH, Sandall J. Threatened preterm labour: women's experiences of risk and care management: a qualitative study. Midwifery. 2018;64:85-92.

29. NHS England. A Saving Babies Lives Care Bundle Version 2. 2019. https:// www.england.nhs.uk/wp-content/uploads/2019/03/Saving-Babies-LivesCare-Bundle-Version-Two-Final-Version2.pdf.

\section{Publisher's Note}

Springer Nature remains neutral with regard to jurisdictional claims in published maps and institutional affiliations.
Ready to submit your research? Choose BMC and benefit from:

- fast, convenient online submission

- thorough peer review by experienced researchers in your field

- rapid publication on acceptance

- support for research data, including large and complex data types

- gold Open Access which fosters wider collaboration and increased citations

- maximum visibility for your research: over 100M website views per year

At BMC, research is always in progress.

Learn more biomedcentral.com/submissions 